\title{
Nonradial Modes in the Galactic Bulge RR Lyrae Stars
}

\author{
P. Moskalik
}

Copernicus Astron. Center, ul. Bartycka 18, 00-716 Warsaw, Poland

\begin{abstract}
OGLE-1 photometry of RR Lyrae stars has been analyzed in search of multiperiodic pulsations. A second periodicity corresponding to a nonradial mode has been found in $11 \mathrm{RRab}$ and $2 \mathrm{RRc}$ variables.
\end{abstract}

\section{Introduction}

For years the $\mathrm{RR}$ Lyrae variables have been considered a primary example of purely radial pulsators. In a recent paper (Olech et al. 1999) the presence of nonradial modes was demonstrated in 3 RRc stars of M55. This intriguing result has prompted us to conduct a systematic search of secondary periodicities in the RR Lyrae variables of the Galactic bulge, using the photometric data accumulated by the OGLE-1 project (Udalski et al. 1997 and references therein).

\section{Results}

The OGLE-1 database contains $66 \mathrm{RRc}$ (first overtone) and $149 \mathrm{RRab}$ (fundamental mode) variables. From this sample we have excluded 10 RRab stars with obvious Blazhko effect. For the remaining objects, $I$-band photometry has been phased with the primary period $P_{1}$, and then fitted with the Fourier sum. The period $P_{1}$ has also been refined in the fitting process. In the next step, the residuals of the fit have been searched for additional periodicities with the standard techniques (Fourier power spectrum or $\chi^{2}$ periodogram). The nominal frequency resolution of the periodogram is $1 / T=0.0013 \mathrm{~d}^{-1}$ or better.

We have found a clear signature of a secondary period in $15 \mathrm{RR}$ Lyrae stars. In Fig. 1 we display $1 / \chi^{2}$ periodograms of two objects, computed after removing the primary pulsation. The arrow indicates where the primary frequency has been located.

The main results of our search can be summarized as follows:

- We have found one "standard" double-mode or RRd variable: BW7/V30. Its period ratio $P_{1} / P_{2}=0.7435$ is very typical for its class and corresponds to the ratio of radial first overtone to the radial fundamental mode.

- In one star, BW6/V29 (see Fig. 1) we have found two secondary frequencies. Together with the primary frequency, they form an equidistant triplet with the splitting of $\Delta f=0.0227 \mathrm{~d}^{-1}$. The amplitudes of both side-peaks are almost equal. This pattern represents the case of strictly periodic, $\pm 7 \%$ amplitude modulation of the primary pulsation, with $P_{\bmod }=44.0 \mathrm{~d}$. 


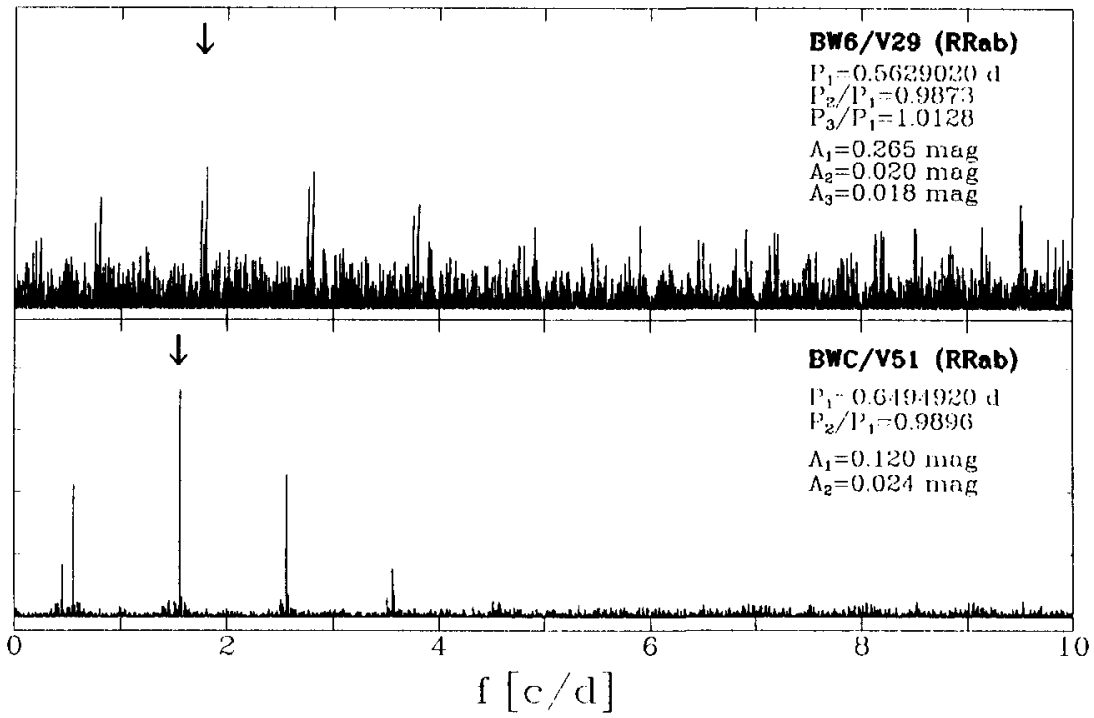

Figure 1. 1/ $\chi^{2}$ periodograms for multimode OGLE-1 RR Lyrae variables. The data have been prewhitened with the primary frequency and its harmonics. The arrow indicates the position of the (removed) primary frequency.

- In the remaining 13 stars we have found a single secondary period, $P_{2}$. The period ratio, $P_{2} / P_{1}$, is in the range of $0.9537-1.0171$. Such a period ratio cannot be explained with two radial modes. Thus, at least one of the periods (presumably the secondary one) corresponds to a nonradial mode.

- For the first time we have found nonradial modes in the RRab variables. The phenomenon is quite common; it occurs in 11 objects, i.e. in $8 \%$ of our RRab sample. It is neither limited to, nor does it favor, any particular range of fundamental mode periods; it occurs for $P_{1}$ from $0.37 \mathrm{~d}$ to $0.68 \mathrm{~d}$.

- We have found nonradial modes in $2 \mathrm{RRc}$ variables. This constitutes $3 \%$ of the studied RRc sample.

- For both RRc variables, the period ratio $P_{2} / P_{1}>1$. The opposite tendency is shown by the RRab stars: 9 out of 11 objects have period ratios in the narrow range of $0.9853-0.9929$.

- The semi-amplitude of the secondary mode is always very small, never above $0.05 \mathrm{mag}$. It tends to be smaller in the RRab variables. While the primary pulsation is always asymmetric, the secondary mode is perfectly sinusoidal for all stars.

\section{References}

Olech, A., Katużny, J., Thompson, I. B., et al. 1999, AJ, 118, 441

Udalski, A., Olech, A., Szymański, M., et al. 1997, Acta Astron., 47, 1 